\title{
Update in Hospital Palliative Care: Symptom Management, Communication, Caregiver Outcomes, and Moral Distress
}

\author{
Rachel D. Havyer, MD', Daniel H. Pomerantz, MD, MPH², Robert L. Jayes, MD³, \\ Patricia F. Harris, MD, MS', Stephanie M. Harman, MD, Aziz A. Ansari, DO6*
}

\begin{abstract}
${ }^{1}$ Division of Primary Care Internal Medicine and Center for Palliative Medicine, Mayo Clinic, Rochester, Minnesota; ${ }^{2}$ Division of General Internal Medicine and Department of Family Medicine (Palliative Care), Albert Einstein College of Medicine, Bronx, New York, and Department of Medicine, Montefiore New Rochelle Hospital, New Rochelle, New York; ${ }^{3}$ Division of Geriatrics and Palliative Medicine, George Washington University Medical Faculty Associates, Washington, D.C.; ${ }^{4}$ Division of Geriatrics, Department of Medicine, David Geffen School of Medicine, University of California, Los Angeles; ${ }^{5}$ Department of Medicine, School of Medicine, Stanford University, Stanford, California; ${ }^{6}$ Division of Hospital Medicine, Loyola University Medical Center, Maywood, Illinois.
\end{abstract}

BACKGROUND: Updated knowledge of the palliative care $(\mathrm{PC})$ literature is needed to maintain competency and best address the PC needs of hospitalized patients. We critiqued the recent PC literature with the highest potential to impact hospital practice.

METHODS: We reviewed articles published between January 2016 and December 2016, which were identified through a handsearch of leading journals and a MEDLINE search. The final 9 articles selected were determined by consensus based on scientific rigor, relevance to hospital medicine, and impact on practice.

RESULTS: Key findings include the following: scheduled antipsychotics were inferior to a placebo for nonterminal delirium; a low-dose morphine was superior to a weak opioid for moderate cancer pain; methadone as a coanalgesic improved high-intensity cancer pain; many hospitalized patients on comfort care still receive antimicrobials; video decision aids improved the rates of advance care planning (ACP) and hospice use and decreased costs; standardized, PC-led intervention did not improve psychological outcomes in families of patients with a chronic critical illness; caregivers of patients surviving a prolonged critical illness experienced high and persistent rates of depression; people with nonnormative sexuality or gender faced additional stressors with partner loss; and physician trainees experienced significant moral distress with futile treatments.

CONCLUSIONS: Recent research provides important guidance for clinicians caring for hospitalized patients with serious illnesses, including symptom management, $A C P$, moral distress, and outcomes of critical illness. Journal of Hospital Medicine 2018;13:419-423. Published online first December 20, 2017. () 2018 Society of Hospital Medicine. he aim of palliative care (PC) is to improve quality of life for patients facing serious, life-threatening illness and their families. ${ }^{1}$ Due to insufficient numbers of PC specialists to meet the PC needs for every hospitalized patient, ${ }^{2}$ all hospitalists should maintain basic PC skills as recognized by PC being a core competency for hospitalists. ${ }^{3,4}$

We summarize and critique PC research articles published between January 1, 2016, and December 31, 2016, that have a high likelihood of impacting the practice of hospital medicine. We hand searched 15 journals and conducted a MEDLINE keyword search of PC terms (see Table). All titles and/or abstracts were reviewed and selected for full review based on the following factors: palliative medicine content, scientific rigor, im-

*Address for correspondence: Aziz A. Ansari, DO, 2160 S. First Ave, Maywood, IL 60153; Telephone: 708-216-5118; Fax: 708-216-8188; E-mail: aansar1@lumc.edu

Received: May 30, 2017; Revised: August 9, 2017;

Accepted: September 15, 2017

2018 Society of Hospital Medicine DOI 10.12788/jhm.2895 pact on practice, and relevance to hospital medicine. Fifty-five articles were individually reviewed and scored by all authors according to rigor, impact, and relevance. Articles were ranked according to their mean scores, and 9 articles were chosen for inclusion through consensus discussion.

\section{SYMPTOM MANAGEMENT}

Antipsychotics Were Inferior to a Placebo in Treating Nonterminal Delirium

Agar MR, Lawlor PG, Quinn S, et al. Efficacy of oral risperidone, haloperidol, or placebo for symptoms of delirium among patients in palliative care: a randomized clinical trial. JAMA Intern Med. 2017;177(1):34-42.

\section{Background}

Delirium is highly prevalent in PC and is associated with significant distress. ${ }^{5}$ Antipsychotics are widely used for symptoms of delirium, although current evidence does not support this practice in hospitalized adults.,

\section{Findings}

This was a double-blind, parallel-arm, placebo randomized 


\begin{tabular}{l}
\hline TABLE. Fifteen Journals Included in Hand Search \\
for Palliative Care Studies Impacting Hospital Medicine \\
Practice \\
\hline American Journal of Hospice and Palliative Care \\
Annals of Internal Medicine \\
British Medical Journal \\
Journal of the American Geriatrics Society \\
Journal of the American Medical Association (JAMA) \\
JAMA Internal Medicine \\
Journal of Clinical Oncology \\
Journal of General Internal Medicine \\
Journal of Hospital Medicine \\
Journal of Pain and Symptom Management \\
Journal of Palliative Medicine \\
Lancet \\
New England Journal of Medicine \\
PC-FACS (Fast Article Critical Summaries for Clinicians in Palliative Care)
\end{tabular}

Search strategy for review of palliative care studies impacting hospital medicine practice

Medline search for English-language articles published between January 1, 2016, and December 31, 2016

Palliative

Pain

End of life

Symptom management

Communication

Hospice

Terminal illness

Advance directives

\section{Implications}

Avoid scheduled antipsychotics in patients with nonterminal delirium, as they can increase risk of harm without advantages, over nonpharmacologic interventions.

Low-Dose Morphine Was Superior to Weak Opioids in the Treatment of Moderate Cancer Pain

Bandieri E, Romero M, Ripamonti $\mathrm{Cl}$, et al. Randomized trial of low-dose morphine versus weak opioids in moderate cancer pain. J Clin Oncol. 2016;34(5):436-442.

\section{Background}

The World Health Organization guidelines recommend the use of weak opioids (WOs), such as codeine or tramadol, as a sequential step in the management of cancer pain. ${ }^{8}$ This strategy has not been tested against low doses of stronger opioids.

\section{Findings}

In this multicenter, open-label RCT, 240 patients in Italy were randomized and stratified by age ( $<75$ years or $\geq 75$ years) to either the WO group or low-dose morphine (M) group. The primary outcome measure was a reduction in pain intensity by $20 \%$ or more. Secondary outcomes included an improvement in symptom scores, a $\geq 30 \%$ and $\geq 50 \%$ reduction in pain, increased opioid dosage, and adverse side effects. Compared with the WO group, the M group had more patients with a $20 \%$ reduction in pain $(88.2 \%$ vs $54.7 \%$; $P<.001)$, more evidence of pain control in the first week $(80.9 \%$ vs $43.6 \%$; $P<$ .001 ), more patients with $a \geq 30 \%$ and $\geq 50 \%$ reduction in pain, and less need to switch to a stronger opioid (15.5\% vs $35.0 \%$; $P$ $=.001)$ or require dose increases. Adverse effects were similar in both groups.

\section{Cautions}

Patients with chronic kidney disease (CKD) were excluded due to concerns about the accumulation of morphine metabolites. Additionally, this study was open label, increasing the risk of bias.

\section{Implications}

Low-dose morphine should be considered over the use of WOs to achieve better and more rapid pain control in patients without CKD.

The Use of Methadone as a Coanalgesic May Improve Moderate Cancer Pain

Courtemanche F, Dao D, Gagné F, et al. Methadone as a coanalgesic for palliative care cancer patients. J Palliat Med. 2016;19(9):972-978.

\section{Background}

Methadone is effective at treating cancer pain and is often utilized when patients have neuropathic pain, fail to respond to traditional opioids, or have renal failure. ${ }^{9,10}$ However, its long half-life and many drug interactions make methadone challenging to use. 


\section{Findings}

This cohort study looked at 153 inpatient or outpatient PC patients in Montreal who received methadone as a coanalgesic for cancer pain. The patients' median morphine equivalent dose was $120 \mathrm{mg}$ when initiating methadone. The median starting dose of methadone was $3 \mathrm{mg}$ per day. Of patients, $49.3 \%$ had a significant response ( $\geq 30 \%$ pain reduction), with a median response time of 7 days, and $30.1 \%$ achieved a substantial response ( $\geq 50 \%$ pain reduction), with a median response time of 3 days. Patients with higher initial pain scores were more likely to respond to adjuvant methadone. Those who had not responded after a week of methadone were unlikely to respond despite dose escalations. Adverse effects included drowsiness (51.4\%), confusion (27.4\%), constipation (24.7\%), nausea (19.9\%), and myoclonia (16.4\%).

\section{Cautions}

This was an observational study with retrospective data, leading to higher levels of missing data. A high rate of adverse side effects was reported (90.4\%). Further study is needed to validate and reproduce the findings.

\section{Implications}

The use of adjuvant low-dose methadone may be considered in patients with moderate pain despite high-dose opioids. If a response is not seen within 7 days, then methadone use should be reconsidered.

\section{ANTIBIOTIC STEWARDSHIP}

Many Hospitalized Patients on Comfort Care Still Receive Antimicrobials

Merel SE, Meier CA, McKinney CM, Pottinger PS. Antimicrobial use in patients on a comfort care protocol: a retrospective cohort study. J Palliat Med. 2016;19(11):1210-1214.

\section{Background}

It is unknown how often patients who are hospitalized at the end of life continue to receive antimicrobials and what factors are associated with antimicrobial use.

\section{Findings}

This retrospective cohort study of 1881 hospitalized adults transitioned to a comfort care order (CCO) set at 2 academic medical centers found that $77 \%$ of these patients received antimicrobials during their hospital stay $(62.4 \%$ at 24 hours prior to $\mathrm{CCO}$ ). Of the 711 still alive at $\geq 24$ hours after $\mathrm{CCO}$, $111(15.6 \%)$ were still on antimicrobials, with that proportion remaining stable for the remainder of hospitalization. In comparing those who did and did not receive antimicrobials after 24 hours of $\mathrm{CCO}$, the presence of a documented infection was not significantly different after adjusting for age. Those with a cancer diagnosis (adjusted risk ratio $[$ ARR] $=1.44: P=.04$ ), a longer length of stay ( $\geq 7$ days vs $<7$ days; $A R R=1.49 ; P=.05$ ), and those discharged home (ARR 2.93; $P<.001$ ) or to a facility (ARR 3.63; $P<.001$ ) versus dying in the hospital were more likely to be on antimicrobials 24 hours after CCO. Compared with those on a medicine service, patients in the medical and surgical intensive care units (ICUs) were less likely to receive antimicrobials (medical ICU ARR $=0.32 ; P=.01$; surgical ICU and/ or neuro-ICU ARR $=0.32 ; P=.02$ ). The most commonly administered antimicrobials were fluoroquinolones and vancomycin.

\section{Cautions}

Only 111 patients were still on antimicrobials at 24 hours, which limited analysis. Investigators relied on retrospective data for medication administration and diagnoses.

\section{Implications}

Further work is needed to understand and address the expectations of clinicians, patients, and families regarding the role of antimicrobials at the end of life.

\section{COMMUNICATION AND DECISION MAKING}

Video Decision Aids Improved Rates of Advance

Care Planning and Hospice Use and Decreased

Costs

Volandes, AE, Paasche-Orlow MK, Davis AD et al. Use of video decision aids to promote advance care planning in Hilo, $\mathrm{Ha}$ wai'i. J Gen Intern Med. 2016;31(9):1035-1040.

\section{Background}

Advance care planning (ACP) can be enhanced with the use of video decision aids, which may help address scalability and cost. ${ }^{11}$ The Hawaii Medical Service Association began an initiative to improve ACP rates, which included a financial incentive. Clinician training and patient access to ACP videos were implemented 1 year into this campaign, which was intended for patients with late-stage disease.

\section{Findings}

This study tested the impact of the video intervention on the rates of ACP documentation in Hilo, Hawaii, along with secondary outcomes of hospice use, hospital deaths, and costs. The intervention was sequentially rolled out to Hilo Medical Center (HMC), followed by hospice and primary care practices. Following the video introduction, the proportion of patients discharged from HMC with ACP documentation markedly increased (3.2\% to 39.9\%; $P<.001)$. The percentage of hospital patients discharged to hospice increased from $5.7 \%$ to $13.8 \%(P$ $<.001)$. Overall admissions to the Hospice of Hilo increased at a greater rate than in other parts of Hawaii. After the intervention in Hilo, the in-hospital death rate among patients $>65$ years old declined slightly $(P=.14)$, while in the rest of the state, the rate remained essentially unchanged. ACP planning did not reduce healthcare costs at the end of life, but costs seemed to increase more slowly in Hilo after the intervention than they did in the rest of Hawaii $(P<.05)$.

\section{Cautions}

This report relies on before-and-after comparisons, with potential confounding by a background pay-for-quality initiative; however, the timing of the changes in outcomes correlates 
well with the introduction of the videos. ACP videos have been studied in other settings, so the intervention is likely generalizable to other states.

\section{Implications}

A widespread distribution of ACP videos and training for physicians in their use may lead to significant increases in ACP documentation and other beneficial clinical outcomes for patients and health systems.

A Standardized Palliative Care-Led Intervention Did Not Improve Psychological Outcomes in Families of Patients with Chronic Critical Illness

Carson SS, Cox CE, Wallenstein S, et al. Effect of palliative care-led meetings for families of patients with chronic critical illness: a randomized clinical trial. JAMA. 2016;316(1):51-62.

\section{Background}

Chronic critical illness (CCl) occurs when a patient neither recovers nor dies for days to weeks after an acute illness requiring aggressive intensive care. $\mathrm{CCl}$ is associated with poor patient and family outcomes. ${ }^{12}$ Does a protocol-driven support and information meeting led by PC providers improve these outcomes?

\section{Findings}

This multicenter RCT compared $130 \mathrm{CCl}$ patients (184 surrogates) who received a structured intervention to 126 patients (181 surrogates) with usual care. The structured intervention was led by PC clinicians in order to provide supportive conversations and information about $\mathrm{CCl}$ and prognosis compared with the usual intensivist communication. The support and information team met with the families of patients in the intervention group after day 7 of mechanical ventilation (MV) and again 10 days later. Both the intervention and control groups received validated information about $\mathrm{CCl}$, and all were eligible for specialty PC consultation, as indicated. The primary outcome of the study was the Hospital Anxiety and Depression Scale (HADS) at 90-day follow-up with the surrogates. Secondary endpoints included posttraumatic stress disorder (PTSD) assessment and other communication measures as well as patient outcomes (hospital mortality, 90-day survival, length of stay, and days of MV). At least 1 meeting took place for $89 \%$ of patients (82\% of surrogates) in the intervention arm. Fewer patients in the intervention arm had nonstudy PC consultations (13\% vs $22 \%$ ). Ninety-day HADS results were similar in the 2 groups. PTSD symptoms, however, were higher in the intervention group (Impact of Event Scale-Revised score: 25.9 for intervention and 21.3 for control; intergroup difference 4.6 [95\% confidence interval, 0.01-9.10]). There were no statistically significant differences among the patient-focused measures, including survival.

\section{Cautions}

Although the teams contained skilled clinicians led by PC practitioners, this was not an ordinary PC intervention. The intervention included information and emotional support meetings alone rather than support from a PC team driven by clinical considerations. This study included surrogates of patients with $\mathrm{CCl}$ but not other conditions.

\section{Implications}

Protocol-driven support and information meetings may not improve, and may slightly worsen, outcomes in families of patients with $\mathrm{CCl}$. This study did not evaluate and should not be applied to clinically indicated, specialty PC consultation in the ICU.

\section{CAREGIVER OUTCOMES}

Caregivers of Patients Surviving Prolonged Critical Illness Experience High and Persistent Rates of Depression

Cameron JI, Chu LM, Matte A, et al. One-year outcomes in caregivers of critically ill patients. N Engl J Med. 2016;374(19):18311841.

\section{Background}

More than half of patients with a $\mathrm{CCl}$ require caregiver support 1 year after hospitalization. ${ }^{13}$ Caregivers provide tremendous physical and psychosocial support to their loved ones, but that care is often associated with significant burden. ${ }^{14}$

\section{Findings}

This prospective parallel cohort study followed caregivers of surviving patients ventilated for at least 7 days from 10 academic hospitals in Canada. The prevalence of depression (Center for Epidemiologic Studies-Depression scale $\geq 16$ ) in this cohort of 280 caregivers (70\% were women) was $67 \%, 49 \%, 43 \%$, and $43 \%$ at the survey intervals of 7 days, 3 months, 6 months, and 12 months after ICU discharge, respectively. Using latent-class linear mixed models, the investigators identified 2 groups of caregivers: those whose depressive symptoms decreased over time (84\%) and those whose depressive symptoms persisted at a high level for the year (16\%). Patient characteristics (such as age, comorbidity, sex, and functional status) were not associated with caregiver outcomes. Younger caregiver age, greater effect of patient care on other activities, less social support, less mastery (sense of control), and less personal growth were associated with worse caregiver mental health outcomes.

\section{Cautions}

Although this is a high-quality prospective study, causality of caregiving on the high rates of depressive symptoms cannot be confirmed without a control group or knowledge of the caregivers' mental health status prior to the episode of prolonged critical illness.

\section{Implications}

Patient critical illness may have serious impacts on caregiver health and well-being. Hospitalists should be attentive to factors associated with caregiver vulnerability and offer support. Improving caregivers' sense of control and social support may be targets for interventions. 


\section{People with Non-normative Sexuality or Gender} Face Additional Barriers and Stressors with Partner Loss

Bristowe K, Marshall S, Harding R. The bereavement experiences of lesbian, gay, bisexual and/or trans* people who have lost a partner: A systematic review, thematic synthesis and modelling of the literature. Palliat Med. 2016;30(8):730-744.

\section{Background}

Grief and bereavement impact individuals differently as they adjust to a death. Increasingly, it is recognized that lesbian, gay, bisexual, and/or transgender (LGBT) communities may face additional barriers when interacting with the healthcare system. This review sought to identify and appraise the evidence of the bereavement experiences among LGBT communities.

\section{Findings}

This systematic review summarized quantitative and qualitative data from 23 articles (13 studies). The synthesis noted that the pain associated with the loss of a partner was a universal experience regardless of sexual identity or gender history. Additional barriers and stressors of bereavement were reported for LGBT people, including homophobia, failure to acknowledge the relationship, additional legal and financial issues, and the shadow of human immunodeficiency virus (HIV) or acquired immunodeficiency syndrome (AIDS). LGBT people turned to additional resources for bereavement help: professional support, social and familial support, and societal and community support. Caregiver bereavement support experiences were shaped by whether the relationships were disclosed and accepted (acceptance-disclosure model).

\section{Cautions}

The quantitative data was mostly from the 1990s and described the context of HIV/AIDS. The qualitative studies, however, were done in the last decade. Very little research was available for transgender or bisexual caregivers.

\section{Implications}

People who identify as LGBT face additional barriers and stressors with the loss of a partner. The described acceptance-disclosure model may help providers be mindful of the additional barriers to LGBT bereavement support.

\section{MORAL DISTRESS AND RESILIENCY}

Physician Trainees Experience Significant Moral Distress with Futile Treatments

Dzeng E, Colaianni A, Roland M, et al. Moral distress amongst American physician trainees regarding futile treatments at the end of life: a qualitative study. J Gen Intern Med. 2016;31(1):93-99.

\section{Background}

Physician trainees are often faced with ethical challenges in providing end-of-life care. These ethical challenges can create confusion and conflict about the balance between the benefits and burdens experienced by patients.

\section{Findings}

The authors used semistructured, in-depth, qualitative interviews of 22 internal medicine trainees from 3 academic medical centers. An analysis of these interviews revealed several themes. Trainees reported moral distress when (1) many of the treatments provided in end-of-life care (ie, feeding tubes in advanced dementia) were perceived to be futile; (2) they felt obligated to provide end-of-life care that was not in the patient's best interest, leading to "torture" or "suffering" for the patient; (3) they provided care they felt not to be in the patient's best interest; (4) they perceived themselves to be powerless to affect change in these dilemmas; (5) they attributed some of their powerlessness to the hierarchy of their academic institutions; and (6) they feared that dehumanization and cynicism would be required to endure this distress.

\section{Cautions}

Resident recruitment occurred by solicitation, which may invite bias. Generalizability of qualitative studies to other settings can be limited.

\section{Implications}

Trainees may experience several dimensions of moral distress in end-of-life care. These findings challenge training programs to find ways to reduce the dehumanization, sense of powerlessness, and cynicism that this distress may cause.

Disclosure: The authors declare that they have no relevant financial conflicts of interest.

\section{References}

1. Morrison RS, Meier DE. Palliative care. N Engl J Med. 2004;350:2582-2590.

2. Quill TE, Abernethy AP. Generalist plus specialist palliative care—creating a more sustainable model. N Engl J Med. 2013;368(13):1173-1175.

3. Meier DE. Palliative care in hospitals. J Hosp Med. 2006;1:21-28.

4. Society of Hospital Medicine. Palliative care. J Hosp Med. 2006;1,S1:80-81.

5. Hosie A, Davidson PM, Agar M, Sanderson CR, Phillips J. Delirium prevalence, incidence, and implications for screening in specialist palliative care inpatient settings: a systematic review. Palliat Med. 2013;27(6):486-493.

6. Carnes M, Howell T, Rosenberg M, Francis J, Hildebrand C, Knuppel J. Physicians vary in approaches to the clinical management of delirium. J Am Geriatr Soc. 2003;51(2):234-239.

7. Neufeld KJ, Yue J, Robinson TN, Inouye SK, Needham DM. Antipsychotic medication for prevention and treatment of delirium in hospitalized adults: a systematic review and meta-analysis. J Am Geriatr Soc. 2016;64(4):705-14.

8. WHO. Cancer Pain Relief. 2nd ed. Geneva, Switzerland: WHO; 1996.

9. Leppert $W$. The role of methadone in cancer pain treatment-a review. Int J Clin Pract. 2009;63(7):1095-1109.

10. Morley JS, Bridson J, Nash TP, et al. Low-dose methadone has an analgesic effect in neuropathic pain: a double-blind randomized controlled crossover trial. Palliat Med. 2003;17(7):576-587.

11. Institute of Medicine. Dying in America: Improving Quality and Honoring Individual Preferences Near the End of Life. Washington, DC: The National Academies Press; 2014

12. Nelson JE, Cox CE, Hope AA, Carson SS. Chronic Critical Illness. Am J Respir Crit Care Med. 2010;182(4):446-454.

13. Chelluri L, Im KA, Belle SH, et al. Long-term mortality and quality of life after prolonged mechanical ventilation. Crit Care Med. 2004;32(1):61-9.

14. Van Beusekom I, Bakhshi-Raiez F, deKeizer NF, Dongelmans DA, van der Schaaf M. Reported burden on informal caregivers of ICU survivors: a literature review. Crit Care. 2015;20:16. 